\title{
LA ORIENTACIÓN AL MERCADO EN LAS MICROEMPRESAS DE LA CIUDAD DE MEDELLÍN COMO MEDIDA PARA INDENTIFICAR LA COMPETITIVIDAD
}

\author{
market orientation in micro-enterprises in the city of medellín as a measure to \\ identify competitiveness
}

Laura Isabel Londoño Guerrero lalondono4@poligran.edu.co

Darya Valentina Arismendi Barrera daarismendi@poligran.edu.co

Institución Universitaria Politécnico Grancolombiano Mercadeo y Publicidad

Colombia

Resumen

Problema de investigación abordado: De acuerdo con un informe presentado por Confecámaras, durante el primer trimestre del año 2018, cerca de 48.052 empresas cerraron sus puertas en Colombia. Esta situación también es expuesta por Romero (2018) donde se enuncia que, más del 95\% de las organizaciones en Colombia son microempresas y que, a su vez, se enfrentan con bajas posibilidades de crecimiento, poca fuerza de negociación y difícil manejo de economías de escala; lo que puede traducirse en un bajo nivel de competitividad.

Objetivo del estudio: Identificar el grado de orientación al mercadeo de las Microempresas de la ciudad de Medellín como medida que refleja el grado de competitividad en el mercado.

Objetivos específicos: 1 . Describir características de las Microempresas presentes en la ciudad de Medellín. 2. Identificar los constructos de mayor influencia con respecto al grado de orientación al mercadeo. 3. Especificar los sectores donde las Microempresas pueden ser más competitivas a partir de la orientación al mercado.

Metodología: Se trabajó con un muestreo por conveniencia aplicando la escala MARKOR de Kohli, Jaworski y Kumar (1993) donde se miden tres dimensiones: 1. Inteligencia de Mercado; 2. Difusión interna de la información de mercado; 3. Respuesta de la empresa hacia el mercado. El instrumento se aplicó a 25 personas que desempeñaban cargos directivos o eran dueños de las organizaciones.

Resultados: El análisis de la información arrojó que los constructos 'Generación de información sobre el Mercado' y ‘Capacidad de respuesta de la empresa' son aquellos que se relacionan en mayor grado con respecto al nivel de orientación al mercado. Finalmente, se encuentra que el sector 'Automotriz' y 'Tecnología' son quienes están más orientados al mercado y, por ende, son más competitivos.

Palabras clave:

Orientación al mercado, competitividad, microempresa. 


\section{INTRODUCCIÓN}

El músculo empresarial de la ciudad de Medellín está conformado principalmente por microempresas; de acuerdo con el portal medellincomovamos.org, para el año 2015 "el 87\% eran microempresas, el 9,6\% pequeñas empresas, el 2,6\% empresas medianas y el 0,8\% empresas grandes, conservando una estructura similar a la de 2012" (párr. 5). De acuerdo con esto, se hace imperativo el que se puedan crear escenarios y medidas que propicien la permanencia, crecimiento y competitividad de las microempresas. Sin embargo, de acuerdo con un informe presentado por Confecámaras, durante el primer trimestre del año 2018, cerca de 48.052 empresas cerraron sus puertas en Colombia. Esta situación también es expuesta por Romero (2018) donde se enuncia que, más del 95\% de las organizaciones en Colombia son microempresas y que, a su vez, se enfrentan con bajas posibilidades de crecimiento, poca fuerza de negociación y difícil manejo de economías de escala; lo que puede traducirse en un bajo nivel de competitividad(Escobar Castro et al., 2020).

A partir de lo anterior, se considera la necesidad de realizar un diagnóstico del grado de competitividad de las microempresas, haciendo uso del concepto Orientación al Mercado que, como lo expresa Rivera (1995) - citado por Bigné (2010) en su obra ‘Raíces y evolución conceptual del Marketing’ - es:

"una estrategia competitiva que desarrolla una organización para alcanzar sus objetivos de negocios. Esta estrategia supone que la satisfacción de los mercados genera una posición competitiva de diferenciación, y que esta posición se traduce en una performance de negocios en el largo plazo".

\section{Orientación al mercado}

La orientación al mercado es un concepto que se asocia a beneficios para las organizaciones; entre ellos, se encuentra la competitividad. De acuerdo con Solano \& Castellanos (2017):

"Se evidencia de manera universal la relación positiva existente entre la orientación al mercado y los resultados de las empresas. Las empresas que de una u otra forma logran conocer y actuar sobre su mercado tienen un desempeño superior, creando una ventaja competitiva sobre sus rivales. La orientación al mercado es una capacidad desarrollada por las empresas para enfrentar de manera exitosa la competencia, los gustos cambiantes y cada vez más exigentes de sus compradores, la globalización y los rápidos cambios en la economía local y mundial. La cultura organizacional y los procesos operativos requeridos en el desarrollo de la orientación al mercado como una capacidad estratégica dan a las empresas".

(p. 92)

En este orden de ideas, la orientación al mercado puede ser definida como los esfuerzos que realizan las organizaciones para la constante creación de valor en los mercados objetivos, contemplando siempre la información de mercado (Coca, A. 2008). Narver y Slater (1990) asimismo, la definieron como la cultura organizativa que más eficaz y eficientemente crea los comportamientos necesarios para la provisión de un valor superior para los compradores y que consiste en tres componentes de comportamiento: orientación al cliente, orientación al competidor y coordinación interfuncional, citado por Álvarez González, Santos Vijande, \& Vázquez Casielles (2005. p. 162). Por otra parte, Kohli, Jaworski, \& Kumar (1993), lo definen a través del uso de tres categorías en su escala MARKOR, a saber: generación de información sobre el mercado, diseminación de la información de la empresa y capacidad de respuesta de la empresa. 


\section{Escalas de medición}

\section{- Escala de Narver y Slater (1990)}

También conocida como escala MKTOR es considerada una de las escalas más importantes para la definición de la percepción cultural de la orientación al mercado. Sus autores la dividen en tres dimensiones: orientación al cliente, orientación al competidor y coordinación interfuncional, en la cual se encuentran 15 items repartidos en cada una, rigiéndose por las perspectivas de rentabilidad y tiempo (Narver \& Slater, 1990).

\section{- Escala de Ruekert (1992)}

La escala de Ruekert, se basa en las acciones que debe tomar la empresa para dejar la filosofía empresarial a un lado y actuar frente al mercado en el que se desarrolla, por eso es apreciada para la estimación de carácter operativo, volviéndola fiable para diferentes organizaciones y negocios que la aplican. El autor fue inspirado por estudios posteriores de Shapiro, los cuales le ayudaron a definir su escala que se centra en 23 items, situados dependiendo de los constructos que son: Obtención y uso de información sobre los clientes, Desarrollo de la estrategia de orientación al mercado y Puesta en práctica de la estrategia de orientación al mercado (Ruekert, 1992).

\section{- Escala de Diamantopoulos y Hart (1993)}

Desarrollan esta escala con el objetivo que sea de estimación, realizando 2 escalas diferentes que se complementan entre sí, teniendo en cuenta que la primera escala es sobre el marketing de manera filosófica y cultural separando sus 12 items en: propósito de marketing o el marketing como hilo conductor de los negocios, concentración en el mercado, papel central de la función de marketing en toda la organización y orientación al cliente. Mientras que la segunda trata al marketing desde la perspectiva operativa, teniendo también 12 items en los siguientes constructos: Generación de información sobre el mercado, Diseminación de la información en la empresa y Capacidad de respuesta de la empresa (Diamantopoulos \& Hart, 1993).

\section{- $\quad$ Escala de Kohli, Jaworski y Kumar (1993)}

Estos autores crean la escala denominada MARKOR que al igual que Nerves y Slater es una de las favoritas para poner en práctica en el campo operativo del Marketing, teniendo la caracterización de orientación al mercado divididas en las siguientes dimensiones: La generación de inteligencia del mercado, Su diseminación interna y El desarrollo y puesta en práctica de una acción de respuesta dirigida al mercado. A diferencia de la escala de MKTOR, la de MARKOR se centra más en el mercado que en el cliente (Kohli, Jaworski, \& Kumar, 1993).

\section{- Escala de Deshpandé, Farley y Webster (1993)}

Esta escala cobra relevancia en su unidad de análisis, más conocida como Quadrad. Dado esto, se propone que al momento de analizar la escala no solo incluya a los miembros de la organización, sino también tiene en cuenta al cliente (Deshpandé, Farley, \& Webster, 1993). Los constructos que propone analizar esta escala son: 1. Medir de forma rutinaria y regular al cliente; 2. Desarrollar productos y servicios basados en información idónea del mercado y de los clientes; 3. Conocer bien a los competidores; 4 . Saber cómo los clientes valoran los productos y servicios; 5 . Orientarse más a los clientes que los competidores; 6. Competir en función de la diferenciación de los productos o servicios; 7. El primer aspecto por considerar por encima del de los propietarios, son los clientes; 8. Los productos/servicios son los mejores en este negocio; 9. Pensar que el negocio existe principalmente para servir a los clientes.

\section{- Escala de Pelham (1993)}

Esta escala se enfoca en la orientación cultural al mercado. Su unidad de análisis tiene preeminencia en las pequeñas empresas de la industria más que en las empresas medianas o grandes (Pelham, 1993). Los constructos de esta escala son: la orientación a la compresión del cliente, la orientación a la 
satisfacción del cliente y la orientación al competidor(Benítez, Quintero, Márquez, \& Ayala, 2015; Díaz, 2016b; Hernández, 2016).

\section{- Escala de Deng y Dart (1994)}

Esta toma en cuenta empresas de distintos tamaños, sectores y localizaciones (Deng \& Dart, 1994). Los constructos bajo los cuales trabaja son: orientación al cliente (ocho subdivisiones), orientación a la competencia (seis subdivisiones), coordinación interfuncional (seis subdivisiones), énfasis en el beneficio (cinco subdivisiones).

\section{- Escala de Deshpandé y Farley (1996)}

Esta se crea con una visión amplia, aplicable a empresas casi de todo tipo de entorno tanto geográfico como industrial - sectores - (Farley \& Deshpandé, 1996). Su esquema es presentado bajo diez aspectos: 1. Objetivos de nuestro negocio orientados principalmente por la satisfacción del cliente; 2. Nivel de compromiso y orientación para servir las necesidades de los clientes; 3 . Información sobre las experiencias (éxito y fracaso) con los clientes a todas las áreas funcionales; 4. Estrategia dirigida a obtener una ventaja competitiva basada en la comprensión de las necesidades de los clientes; 5. Medición de la satisfacción del cliente; 6(Castro \& García, 2016; Díaz, 2016a; Oviedo \& Silva, 2017). Medición de forma rutinaria y regular el servicio al cliente 7. Mayor orientación al cliente que el competidor 8. Pensamos que este negocio existe principalmente para servir a nuestros clientes 9. Contactamos con los clientes finales al menos una vez al año para valorar la calidad de nuestros productos y servicios 10. Los datos sobre la satisfacción del cliente son difundidos a todos los niveles en cada unidad de negocio de un modo regular(Arias-Velandia William Umar; Moreno-Jiménez, Yeimy Julieth \& Ossa-Gallego Gisele Eugenia, 2020; Rincón-Báez, Becerra Plaza, Arias-Velandia, \& Durán Becerra, 2018; Luis Martín; Trujillo-Flórez et al., 2018).

\section{- Escala de Bisp, Harmsen y Grunert (1996)}

Esta escala de medición tiene una perspectiva cultural y, a su vez, operativa; en la cual se tiene en cuenta a todos los integrantes que conforman el entorno del negocio y pone al cliente en primer lugar. Adicionalmente, también tiene en cuenta a la competencia (Bisp, Harmsen, \& Grunert, 1996). Los constructos que pretende integrar son: enfoque en la inteligencia del mercado, nivel de actuación, capacidades, organización y apelación a la información.

\section{- Escala de Gray, Matear, Boshoff y Matheson (1998)}

Estos autores no toman como base las escalas de MKTOR ni MARKOR, pues consideran que se enfocan únicamente en grandes industrias (Gray, Matear, Boshoff, \& Matheson, 1998). Sin embargo, las metodologías que implementan en esta escala son tomadas como referentes para el desarrollo de su escala propuesta. Cuenta con 20 ítems, los cuales son divididos en cinco constructos: orientación al cliente, orientación al competidor, coordinación interfuncional, respuesta y perspectiva al beneficio.

\section{- Escala de Becker y Homburg (1999)}

Toma en cuenta los aspectos relativos al mercado buscando un enfoque innovador y complementario, pues a su vez integra un enfoque cultural y operativo (Becker \& Homburg, 1999). Está compuesto por 60 ítems, a su vez divididos en 16 subdimensiones, conformando así cinco grandes constructos: organización, información, planificación, control y recursos humanos.

\section{Método}

El estudio realizado pertenece a una investigación de tipo concluyente - descriptivo con un diseño no experimental. En su desarrollo, se procedió a realizar lo siguiente: selección del tipo de muestreo, recolección y análisis de la información. 
Muestreo

Se empleó un muestreo por conveniencia o no probabilístico que, se dirigió a directivos o empresarios que quisieran participar de manera voluntaria. Lo anterior, debido a las limitaciones en cuanto al acceso y la disposición de la información por parte de las organizaciones. Por consiguiente, la muestra se conformó por un total de 25 empresas que accedieron a responder el cuestionario de manera voluntaria(Daza-Orozco, 2019).

\section{Recolección de Información}

Para medir el grado de orientación al mercado se empleó el instrumento MARKOR, propuesta por Kohli, Jaworski y Kumar (1993) -haciendo uso de una escala Likert representada numéricamente de 1 a 5- lo anterior, dada la facilidad de aplicación y comprensión de las afirmaciones. Los constructos trabajados con el número de preguntas se relacionan a continuación:

\begin{tabular}{|ccc|}
\hline Constructos & $\begin{array}{c}\text { Número de } \\
\text { preguntas }\end{array}$ & $\begin{array}{c}\text { Denominación de } \\
\text { la variable }\end{array}$ \\
$\begin{array}{c}\text { Generación de } \\
\text { información sobre } \\
\text { el Mercado } \\
\text { Diseminación de } \\
\text { la información de } \\
\text { la empresa } \\
\text { Capacidad de } \\
\text { respuesta de la } \\
\text { empresa }\end{array}$ & 6 & $\mathrm{GIM}$ \\
\hline Tabla 2 Relación de preguntas por constructo. Elaboración propia. & $\mathrm{DIE}$ \\
\hline
\end{tabular}

\section{Análisis de la Información}

Para realizar el procesamiento y análisis de la información, se empleó el software RStudio versión 1.1.456. Con respecto de la caracterización de las microempresas estudiadas, se trabajó con organizaciones pertenecientes a sectores como: telecomunicaciones, medios audiovisuales, automotriz, bebidas, gastronómico, retail, servicios en general, tecnología y telecomunicaciones.

\section{Resultados}

De acuerdo con la muestra estudiada, se puede establecer que aquellas pertenecientes a sectores como: tecnología, gastronomía y automotriz; son quienes presentan, hasta el momento, una mayor permanencia en el mercado (Imagen 1).

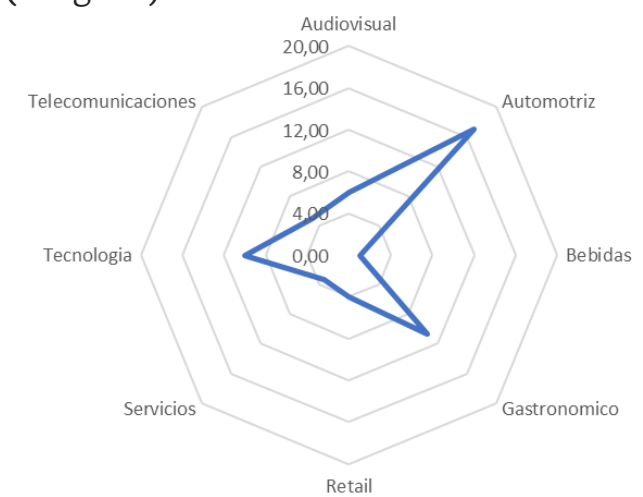

Imagen 1 Permanencia promedio de las microempresas en el mercado por sector. imagen: Elaboración propia. 
En lo referente al grado de orientación, se presenta a continuación la siguiente información:

\begin{tabular}{|c|c|c|c|c|}
\hline Sectores & GIM & DIE & CRE & $\begin{array}{c}\text { Nivel de } \\
\text { Orientación }\end{array}$ \\
\hline Audiovisual & 3,2 & 2,3 & 3,7 & 3,0 \\
\hline Automotriz & 4,5 & 3,9 & 3,4 & 3,9 \\
\hline Bebidas & 2,2 & 2,6 & 3,2 & 2,6 \\
\hline Gastronómico & 3,0 & 3,4 & 3,3 & 3,2 \\
\hline Retail & 3,0 & 3,3 & 3,1 & 3,2 \\
\hline Servicios & 2,5 & 3,3 & 3,1 & 3,0 \\
\hline Tecnología & 4,2 & 3,8 & 3,9 & 3,9 \\
\hline Telecomunicaciones & 3,8 & 3,2 & 3,2 & 3,4 \\
\hline
\end{tabular}

Tabla 2 Resultados por constructo y nivel de orientación. Elaboración propia.

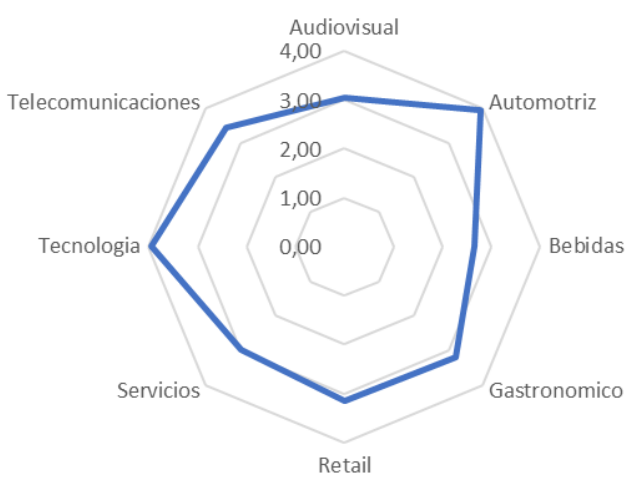

Imagen 2 Grado de orientación al mercado por sector. imagen: Elaboración propia.

De acuerdo con el gráfico anterior, se puede corroborar lo expuesto por Solano \& Castellanos (2017), al afirmar que entre mayor es el grado de orientación al mercado, mayor es la competitividad de la empresa. Esto se ve reflejado al crear un paralelo entre los tres sectores a los que pertenecen las microempresas - de mayor permanencia en el mercado - y aquellas que presentan un mayor grado de orientación al mercado.

Adicionalmente, se identificó el constructo que tiene mayor influencia sobre el nivel de orientación al mercado, encontrándose la siguiente información:

\begin{tabular}{|c|cccc|}
\cline { 2 - 5 } \multicolumn{1}{c|}{} & GIM & DIE & CRE & $\begin{array}{c}\text { Nivel de } \\
\text { Orientación }\end{array}$ \\
\hline GIM & 1 & 0.50 & 0.74 & 0.94 \\
DIE & 0.50 & 1 & 0.32 & 0.66 \\
CRE & 0.74 & 0.32 & 1 & 0.85 \\
NIVEL DE ORIENTACIÓN & 0.94 & 0.66 & 0.85 & 1 \\
GIM & 1 & 0.50 & 0.74 & 0.94 \\
\hline
\end{tabular}

Tabla 3 Correlación de Pearson entre constructos y nivel de orientación al mercado. Elaboración propia.

De acuerdo con la tabla 3, es claro que el constructo 'generación de información sobre el mercado' GIM - es aquel que presenta mayor fuerza (asociación lineal entre las variables) de influencia con respecto a la orientación de mercado (0.94). Esto quiere decir que, si las microempresas que se esfuercen en recopilar información sobre la evolución de las necesidades empleen esta para la toma de decisiones y, especialmente, evalúen periódicamente la percepción de los consumidores con respecto a la calidad de sus productos, son aquellas que podrán permanecer mayor tiempo en el mercado y, por ende, serán más competitivas. 
Expone los hallazgos y datos resultantes del analisis de datos presentados anteriormente en los metodos, los cuales deben tener coherencia y ética respecto al tratamiento de la pregunta de la investigación planteada.

\section{Discusión y Conclusión}

Teniendo en cuenta que la presente investigación se enfocó en identificar los sectores - a los que pertenecen las empresas - con mayor grado de orientación al mercado y, por ende, mayor competitividad, se presenta a continuación, un paralelo entre los resultados obtenidos en la presente investigación con estudios realizados previamente.

\section{Sector Audiovisual}

De acuerdo con los resultados, se puede apreciar que el Sector Audiovisual presenta una débil Orientación al Mercado en comparación con otros; esta situación es similar a los resultados encontrados por Cantillo (2013) en su trabajo sobre su artículo acerca de la incidencia del mercadeo en la competitividad del sector de la comunicación gráfica, donde señala que las empresas que pertenecen a este sector:

"conocen sobre acciones de Marketing y su importancia para ser más competitivas; sin embargo, aceptan que no las aplican con frecuencia debido a no tener una percepción positiva sobre los resultados que el Marketing pueda generar en ellas. En ese sentido, aceptan y reconocen que realizar investigación de mercados, lanzamiento constante de productos nuevos y la planeación estratégica de Marketing dentro de esas organizaciones, es débil".

(p. 145-146).

\section{Sector Servicios}

De la misma manera, los resultados obtenidos en el presente estudio son similares a los hallazgos de Franco, Restrepo \& Sánchez (2014) en su trabajo sobre la gestión del Mercadeo y la competitividad de las pequeñas empresas en Medellín, donde señalan que "no existe una conciencia plena en muchos directivos sobre el aporte de las prácticas de mercadeo a la competitividad de las pequeñas empresas de servicio de Medellín" (p.170).

\section{Sector Tecnología}

Por otra parte, al analizar la influencia de la 'Gestión de la Información de Mercado' sobre la orientación al mercado y, por ende, sobre la competitividad de las pequeñas empresas, se encuentra que los resultados obtenidos difieren de los hallazgos presentados por Gálvez \& Pérez (2011) donde plantean que la cultura de mercado no influye de manera positiva en el rendimiento de las Mipymes.

\section{Conclusiones}

Referente a los constructos, es claro que el de mayor influencia es aquel que se relaciona con la obtención, manejo y aprovechamiento de información del mercado como una fuente para la ventaja competitiva. Sin embargo, no se puede dejar de lado la importancia de que dicha información sea usada y aprovechada por todos los actores de las organizaciones, puesto que:

"está demostrado que se requiere que las personas al interior de las organizaciones establezcan una verdadera relación interfuncional, entre administradores y personal asistencial de manera que venzan las barreras al manejo de la información y sea una estrategia para competir con ventaja y crear una posición fuerte de la institución frente a la competencia".

(Penagos Guzmán, Garavito Culma, \& Pérez Caldón, 2017. p.96). 
Es por lo anterior que el hecho de que, en la presente investigación haya sido el constructo con menor fuerza de asociación con el grado de orientación, no quiere decir que se descuide este aspecto tan importante. Con respecto al grado de orientación al mercado, se pudo encontrar la relación directa que existe entre la permanencia en el mercado y el nivel de orientación al mismo. Adicionalmente, es interesante ver que a pesar de que las microempresas pertenecientes a sectores como tecnología, automotriz y gastronómico son aquellos cuya permanencia y grado de orientación al mercado es mayor en comparación con otros sectores; en general, la orientación al mercado de las microempresas no es tan intensa al afirmar que los resultados promedio no alcanzan a llegar a 4 puntos de 5 . Esto sería atribuible a la baja puntuación obtenida de algunos sectores en el constructo 'generación de información de mercado’ que, como se corroboró anteriormente, es aquel que mayor fuerza de asociación e influencia tiene sobre el grado de orientación al mercado.

\section{Referencias bibliográficas}

Álvarez González, L. I., Santos Vijande, M. L., \& Vázquez Casielles, R. (2005). Escalas de medida del concepto de orientación al mercado. Revisión crítica de su contenido y de sus propiedades psicométricas. Esic market, 161-202.

Becker, J., \& Homburg, C. (1999). Market-Oriented Management: A System-Based Perspective. Journal of Market Focused Management, 17-41.

Benítez, P., Quintero, M., Márquez, K., \& Ayala, A. O. (2015). Beneficios y garantías de los miembros de la fuerza pública en marcos de justicia transicional en perspectiva comparada: lecciones para el caso colombiano. Panorama, 9(16), 105-116. https://doi.org/10.15765/pnrm.vgi16.630

Bigné, E. (2010). Raíces y evolución conceptual del Marketing. Valencia: Universitat de València.

Bisp, S., Harmsen, H., \& Grunert, K. G. (1996). Improving measurement of market orientation $\neg$ An attitude activity based approach. Proceedings of the 25th EMAC Conference, 75-88.

Calidad de vida: Medellín cómo vamos. (29 de septiembre de 2016). Obtenido de https://www.medellincomovamos.org/medellin-mas-competitiva-entre-2012-y-2015-y-con-oportunidades-en-la-gestionde-microempresas/

Cantillo Guerrero, E. (2013). Factores claves en la gestión de mercadeo que inciden en la competitividad del sector de comunicaciones gráficas de la ciudad de Barranquilla,Colombia. Revista EAN, 140-151.

Coca Carasila, A. M. (2008). El concepto de Marketing: pasado y presente. Revista de Ciencias Sociales, $391-412$.

Castro, A. M. P., \& García, M. J. M. (2016). CALIDAD Y FORMACIÓN DEL PROFESORADO NOVEL EN LAS UNIVERSIDADES PÚBLICAS GALLEGAS - Quality and training of new teaching staff in Galician public universities. Panorama, 10(19), 8-17. https://doi.org/10.15765/pnrm.v10i19.830

Daza-Orozco, CE. (2019). Iniciación científica: conceptualización, metodologías y buenas prácticas. Bogotá. Institución Universitaria Politécnico Grancolombiano. Retrieved from http://www.scoif.com/iniciacion-cientifical

Daza-Orozco, CE. (2018). Notas acerca de la investigación en administración de empresas. Revista Fusión. Bogotá. Fundación Universitaria San Mateo. Retrieved from http://cipres.sanmateo.edu.co/index.php/fusion/article/view/38

Deng, S., \& Dart, J. (1994). Measuring market orientation: A multi-factor, multi-item approach. Journal of Marketing Management, 725-742.

Díaz, A. M. (2016a). Evaluación de la propuesta de enseñanza de las áreas de lenguaje y matemáticas en la Institución Educativa San José del Pantano. Panorama, 9(17), 25-39. https://doi.org/10.15765/pnrm.vgi17.789

Díaz, A. M. (2016b). Evaluación del programa Todos a Aprender en la enseñanza de lenguaje y matemáticas en el municipio de Puerto Escondido-Córdoba. Panorama, 10(19), 46-59. https://doi.org/10.15765/pnrm.v10i19.929

Deshpandé, R., Farley, J. U., \& Webster, F. E. (1993). Corporate Culture, Customer Orientation, and Innovativeness in Japanese Firms: A Quadrad. Journal of Marketing, 23-37.

Diamantopoulos, A., \& Hart, S. (1993). Linking market orientation and company performance: preliminary evidence on Kohli and Jaworski's framework. Journal of Strategic Marketing, 93-121.

Escobar Castro, J. A., Gómez Zambrano, R. A., García López, J., Tunjano Pinzón, I. F., Lasso Silva, E. R., Rojas Alvarez, S. P., ... Martínez Rincón, J. A. (2020). Experiencias de innovación educativa - Tomo 4 (1st ed.; Luis Martín Trujillo-Flórez, ed.). Bogotá: Politécnico Grancolombiano.

Farley, J. U., \& Deshpandé, R. (1996). Understanding Market Orientation: A Prospectively Designed Meta-analysis of Three Market Orientation Scales. Massachusetts: Marketing Science Institute.

Franco Restrepo, J. G., Restrepo Restrepo, J. C., \& Sánchez Giraldo, J. C. (2014). La gestión del mercadeo: un aporte a la competitividad de las pequeñas empresas del sector servicios en Medellín. Pensamiento \& Gestión, 150-174.

Gálvez Albarracín, E. J., \& Pérez de Lema, D. G. (2011). Cultura organizacional y rendimiento de las Mipymes de mediana y alta tecnología: un estudio empírico en Cali, Colombia. Cuadernos de administración, 125-145.

Gray, B., Matear, S., Boshoff, C., \& Matheson, P. (1998). Developing a Better Measure of Market Orientation. European Journal of Marketing, 884-903.

Hernández, Y. M. (2016). La Unión Patriótica: memorias para la paz y la democracia. Panorama, 10(18), 27-38. https://doi.org/10.15765/pnrm.v10i18.822

Kohli, A. K., Jaworski, B. J., \& Kumar, A. (1993). MARKOR: A Measure of Market Orientation. American Marketing Association, 467-477. 
Narver, J. C., \& Slater, S. F. (1990). The Effect of a Market Orientation on Business Profitability. Journal of Marketing , 20-35.

Oviedo, L. B., \& Silva, M. C. (2017). LA INVESTIGACIÓN ACCIÓN Y EL APRENDIZAJE POR PROYECTOS EN EL MARCO DEL MODELO PEDAGÓGICO ENSEÑANZA PARA LA COMPRENSIÓN. EXPERIENCIA DEL COLEGIO VISIÓN MUNDIAL EN COMUNIDADES VULNERABLES DE MONTERÍA-Action research and Project Based Learning in ... Panorama, 11(21), 38-51. https://doi.org/10.15765/pnrm.v11i21.1053

Pelham, A. M. (1993). Mediating and Moderating Influences on the Relationship between Marketing Orientation and Performance. Pennsylvania : Thesis in Business Administration, The Pennsylvania State University.

Penagos Guzmán, F., Garavito Culma, D., \& Pérez Caldón, Y. (2017). Orientación al mercado que utilizan las Instituciones Prestadoras de Servicios de Salud (IPS) en Florencia, Caquetá, Colombia. ESCENARIOS: empresa y territorio, 79-102.

Rincón-Báez, W. U., Becerra Plaza, G. E., Arias-Velandia, N., \& Durán Becerra, E. (2018). Inteligencia de Datos en la formación en Administración y Negocios en Colombia 2018 (Vol. 1; Institución Universitaria Politécnico Grancolombiano, ed.). https://doi.org/10.1017/CBO9781107415324.004

Romero, A. (31 de mayo de 2018). Blog de la facultad de Derecho: Universidad Externado de Colombia. Obtenido de Universidad Externado de Colombia Web Site: https://dernegocios.uexternado.edu.co/negociacion/2018-el-ano-en-que-mas-de-48ooo-empresas-se-quebraron-en-todo-el-pais/

Ruekert, R. W. (1992). Developing a market orientation: An organizational strategy perspective. International Journal of Research in Marketing, 225-245.

Solano Arboleda, D., \& Castellanos Ordoñez, G. (2017). Metaanálisis de la relación entre la orientación al mercado y los resultados de la empresa. Estudios Gerenciales, 87-94.

Trujillo-Flórez, Luis Martín;, Martínez Contreras, R. M., Espitia López, H., Rojas Paredes, J. C., Vargas Leguizamón, Y. R., \& Castro Cabal, G. A. (2018). Experiencias de innovación educativa (1st ed.; L. M. Flórez-Trujillo, ed.). Bogotá: Politécnico Grancolombiano. 